\title{
Isotopic Tracers of Abiotic Amino Acid Synthesis
}

\author{
LAURA M CHIMIAK ${ }^{1}$, JOHN EILER ${ }^{2}$, ALEX SESSIONS ${ }^{2}$ \\ AND CARL BLUMENFELD ${ }^{3}$ \\ ${ }^{1}$ Colorado University, Boulder, Geological Sciences \\ ${ }^{2}$ Caltech \\ ${ }^{3}$ Dantari, Inc \\ Presenting Author: laura.chimiak@colorado.edu
}

The search for extraterrestrial life and evidence of its origins often focuses on small biorelevant compounds such as $\alpha$-amino acids. As known life requires these molecules, their presence could aid the formation or provide evidence of life. To ascertain which of these a compound represents - if they were produced biotically or abiotically- isotopic analyses and relative abundances can constrain their synthetic histories.

Compound-specific isotopic analysis of the amino acids and aldehydes from a suite of carbonaceous chondrites suggest that Strecker synthesis produced meteoritic amino acids. Site-specific carbon isotope analyses of alanine from a sample of the Murchison meteorite further support Strecker synthesis from a ${ }^{13} \mathrm{C}$-rich, possibly ISM-sourced, acetaldehyde and ${ }^{13} \mathrm{C}$-poor cyanide. Quantitative interpretation of such data requires values for fractionations associated with amino acid synthesis. We previously reported preliminary experiments examining isotope effects in the Strecker synthesis; here, we provide the first complete experimental study of this chemistry's isotope effects.

Strecker synthesis of alanine begins with equilibration between acetaldehyde, cyanide, and ammonia and 2aminopropanenitrile; it involves a $20 \%$ isotope effect between 2 aminopropanenitrile's ${ }^{13} \mathrm{C}$-depleted amine-bound carbon and acetaldehyde's carbonyl carbon and a 56.6\% isotope effect between 2-aminopropanenitrile's ${ }^{15} \mathrm{~N}$-enriched amine nitrogen and ammonia. Following equilibration, the 2aminopropanenitrile undergoes hydrolysis into alaninamide with a molecular-averaged normal kinetic isotope effect (i.e., faster for ${ }^{12} \mathrm{C}$ than for ${ }^{13} \mathrm{C}$, 'normal KIE') of up to $10 \%$; however, a secondary process (e.g., deamination) enriches 2aminopropanenitrile in ${ }^{13} \mathrm{C}$ by up to $15 \%$ and obfuscates the KIE's value. Subsequent hydrolysis converts alaninamide into alanine with a $15 \%$ normal KIE between alaninamide's amide carbon and alanine's carboxyl carbon.

These data document the mechanistic controls of the sitespecific and molecular-average isotopic signatures of Streckersynthesized alanine that distinguish it from known biological syntheses and enable predictions for other Strecker-synthesized amino acids' isotopic structures. Applying the isotope effects measured here to previously measured amino acid carbon isotope ratios, we infer isotopic contents of aldehyde precursors from early in solar system history, compare them with meteoritic and planetary samples of these compounds, and demonstrate that Strecker synthesis could account for the synthesis of amino acids from aldehydes in CM and CR meteorites. 